J. Gynäkol. Endokrinol. AT 2019 · 29:99-102 https://doi.org/10.1007/s41974-019-00109-8 Online publiziert:5. September 2019 c Der/die Autor(en) 2019

\section{Christoph Brezinka}

Universitätsklinik für gynäkologische Endokrinologie und Reproduktionsmedizin, Medizinische Universität Innsbruck, Innsbruck, Österreich

\title{
Die Gestationsaltersbestimmung mit Ultraschall
}

\begin{abstract}
Die Berechnung eines Geburtstermins (EGT) auf Basis der letzten Regel ist notorisch unverlässlich, vor allem dann, wenn sich die Schwangere nicht erinnern kann. Die Berechnung auf Basis von Ultraschallmessungen ergibt zwar ein scheinbar exaktes Datum, doch auch hier kann man, ohne es zu bemerken, Ungenauigkeiten von einer Woche und mehr festschreiben. Die Festlegung auf einen Geburtstermin mag für bürokratische Fristenläufe und Stichtage wie den Beginn von Mutterschutz und Karenz wichtig sein. Bei der Entscheidung, etwa eine Geburt einzuleiten oder nicht, sollte man sich stets der immanenten Ungenauigkeit des errechneten Termins bewusst sein. Im Zweifel kann es von Vorteil sein, der Genese des Geburtstermins wer den wann, auf welcher Basis, festgelegt hat - bei der Patientin nachzugehen.
\end{abstract}

Seit gut 200 Jahren wird das Alter der menschlichen Schwangerschaft mit der Naegele-Regel bestimmt (•Tab. 1). Diese Regel basiert auf der Prämisse, dass bei allen Frauen die Ovulation und mit ihr die Konzeption 14 Tage nach dem Beginn der Regelblutung stattfindet. Auf der Naegele-Regel beruhen alle Schwangerschaftsrechner, sowohl die kreisförmigen Rechenschieber wie auch die Kalkulatoren in PIA, Astraia und anderen Dokumentationsprogrammen.

Vom errechneten Geburtstermin sind eine Reihe von Dingen abhängig: 8 Wochen vor dem Geburtstermin beginnt in Österreich das gesetzliche Arbeitsverbot, die meisten Personalabteilungen wollen von ihren Mitarbeiterinnen, die eine Schwangerschaft melden, möglichst frühzeitig einen verbindlichen Geburtstermin wissen, auf dessen Basis dann Mutterschutz und Karenz berechnet werden können. Aber auch Fristen für die Screeninguntersuchungen in der Schwangerschaft sowie das empfohlene Gestationsalter, ab denen bei bestimm- ten Risikokonstellationen die Geburt eingeleitet werden soll, hängen stark vom einmal errechneten Geburtstermin $\mathrm{ab}$.

Die auf der Angabe der letzten Regel basierenden Geburtstermine treffen nur in $30 \% \mathrm{zu}$, sind also recht unverlässlich [1]. Die Ovulation findet keineswegs genau am 14. Zyklustag statt, sondern hat eine Streuung vom 9. bis zum 30. Zyklustag [2]. Nur $10 \%$ aller Frauen ovulieren am 14. Zyklustag, der häufigste Tag des Eisprungs ist der 16. Zyklustag [3]. Nicht alle Frauen führen einen Regelkalender, viele können sich an die letzte Regel nicht erinnern und geben, schon allein um das penetrante Nachfragen zu beenden, ein ungefähres Datum an. Dabei wird der 15. eines Monats 2,5-mal häufiger gewählt als jeder andere Tag [4]. Viele muslimische Frauen geben auf die Frage nicht den ersten, sondern den letzten Tag der Regel an, da sie dann die rituelle Waschung Güsül abdesti (türkisch)/غس Ghushl (arabisch) durchführen.
Tab. 1 Naegele-Regel bei spontaner Schwangerschaft und assistierter Reproduktion

Errechneter Geburtstermin = [erster Tag der letzten Regelblutung] + 7 Tage - 3 Monate +1 Jahr

Bei IVF oder Kryotransfer der Blastozyste: den Tag 19 Tage vor dem Transferdatum als Tag der letzten Regel nach Naegele nehmen Bei IVF oder Kryo im Tag-3 Transfer ("cleavage state"): den Tag 17 Tage vor dem Transferdatum als Tag der letzten Regel nach Naegele nehmen
Tab. 2 Grad der Ungenauigkeit der Be-

stimmung des Geburtstermins nach Anga-

ben zur Konzeption [10]

Verlässlichkeit des Geburtstermins nach Konzeptionsmethode

IVF mit bekanntem Punktions\pm 1 Tag und Transfertermin

Ovulationsauslösung + GV zum Optimum

Ovulationsauslösung und intrauterine Insemination

Nur ein GV im Zyklus \pm 3 Tage \pm 3 Tage

Geführter Temperaturkalender oder Ovulationstest
Tab. 3 Eine nach der 26.SSW durchge-

führte Ultraschallbiometrie hat bei der Festlegung des Geburtstermins eine potenzielle Ungenauigkeit von mindestens drei Wochen [10]

Ungenauigkeit des Geburtstermins mit Ultraschall nach Gestationsalter und gemessenen Strukturen

Fruchtsack 5-6 SSW +3-4 Tage

CRL 6-13 SSW $\pm 3-7$ Tage

FL-BPD-FOD 14-20 SSW \pm 10 Tage

FL-BPD-FOD 20-26 SSW 土11-16 Tage

FL-BPD-FOD 26-32 SSW 土21-25 Tage 


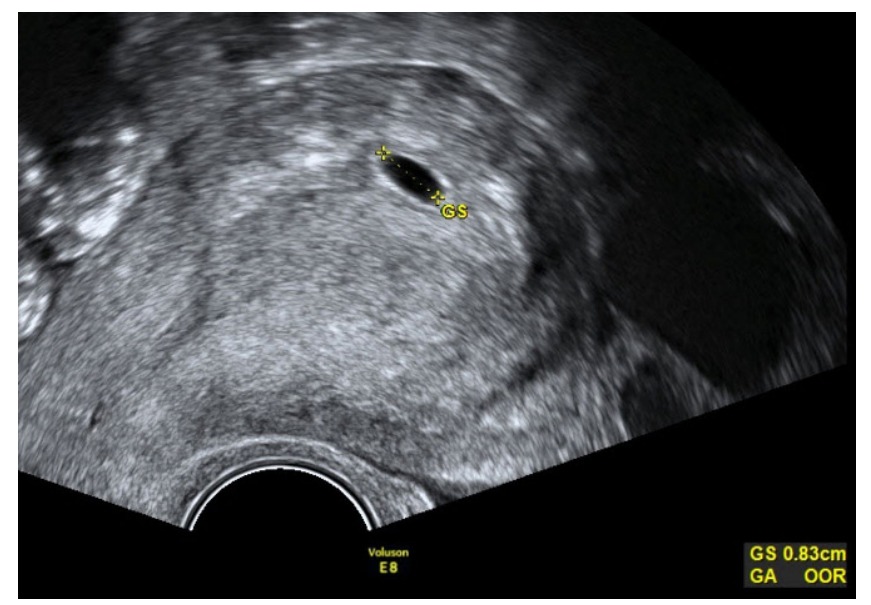

Abb. 1 ム Ein $8 \times 4 \times 7 \mathrm{~mm}$, im Mittel also $6 \mathrm{~mm}$ messender Fruchtsack in der Frühschwangerschaft noch ohne Dottersack und Embryo

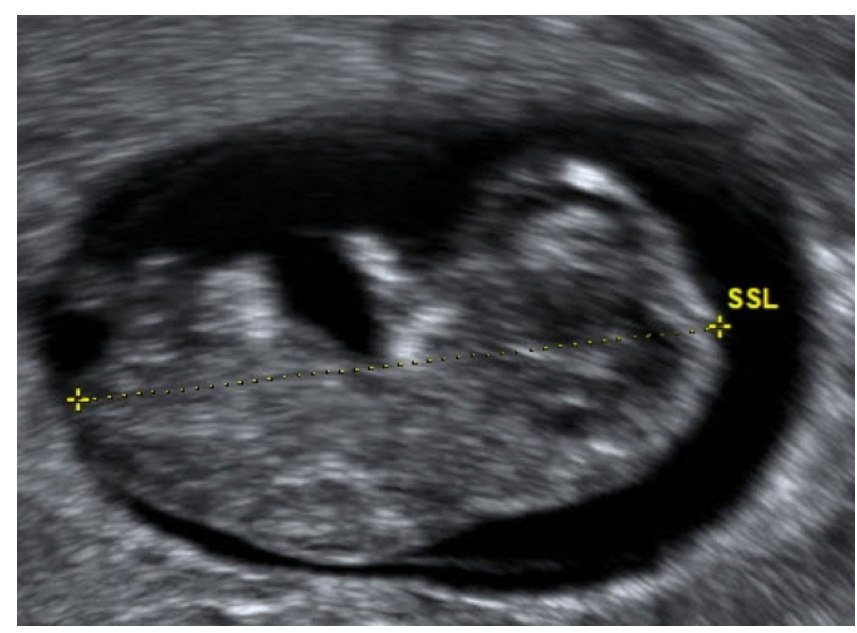

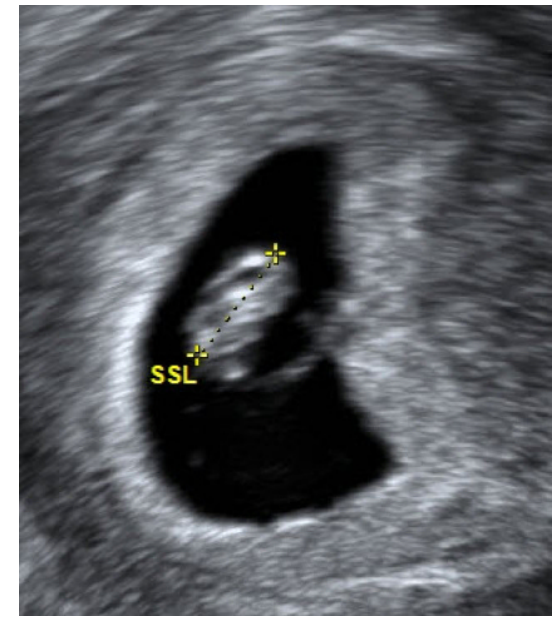

Abb. $5 \Delta 7 \mathrm{~mm}$ langer Embryo, $6+2$ SSW nach der Robinson-Kurve und $6+4$ SSW nach der Pexsters-Kurve
Abb. $3<30 \mathrm{~mm}$ CRL-hier liegen Robinson, Pexsters und Hadlock alle bei $9+5$ SSW

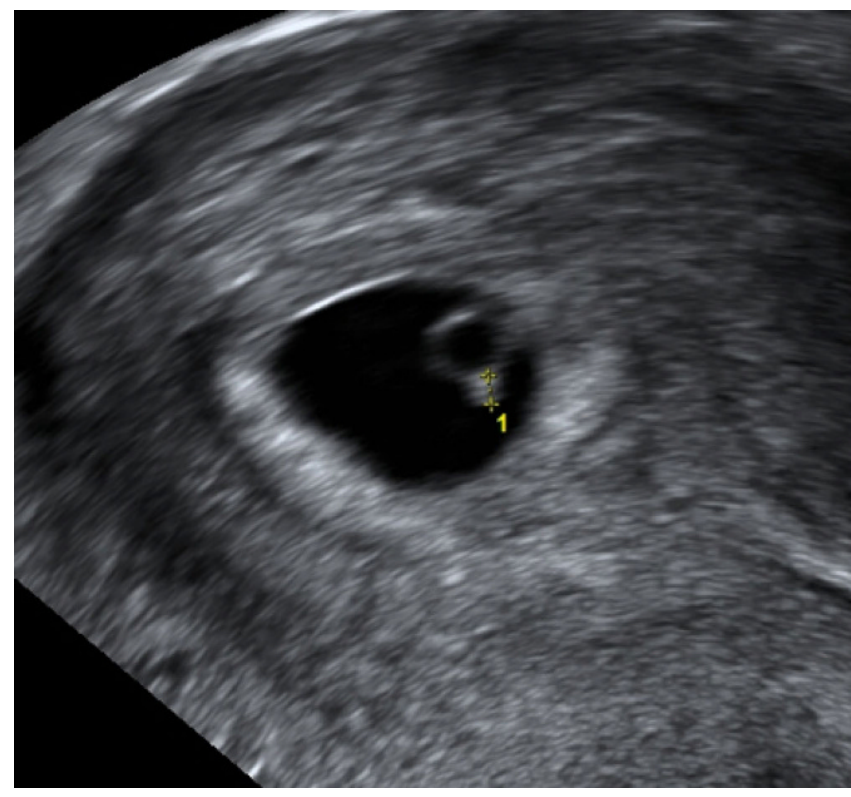

Abb. $2 \Delta$ Erste Zeichen eines Embryos mit 2,2 mm Länge, direkt dem Dottersack anliegend ( $6+0$ SSW nach Pexsters)

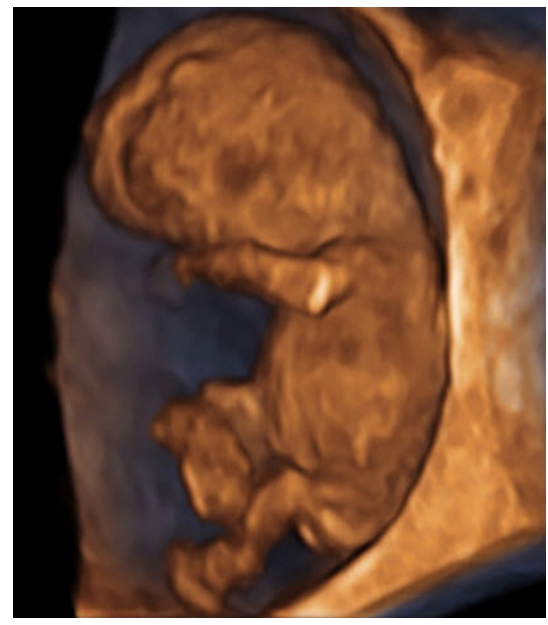

Abb. 4 \ Selber Embryo wie in Abb. 4 im Zuge derselben Untersuchung. 3-D ist optisch wesentlich anschaulicher als B-Mode, allerdings bei Einbringen der Messstrecken wesentlich ungenauer

gel ovulieren [5]. In einer 40 Jahre später mit Vaginalschall durchgeführten belgischen Studie an 3710 normalen Einlingsschwangerschaften zeigte sich, dass die Robinson-Kurve in der 6. SSW das Gestationsalter um 4 Tage unterschätzt (3,7 mm Differenz im CRL), dafür in der 11.-14. SSW um einen Tag $(0,8-1 \mathrm{~mm})$ überschätzt. Die aus dem Jahr 1982 stammenden Hadlock-Kurven unterschätzen das Gestationsalter in der 6. SSW um 


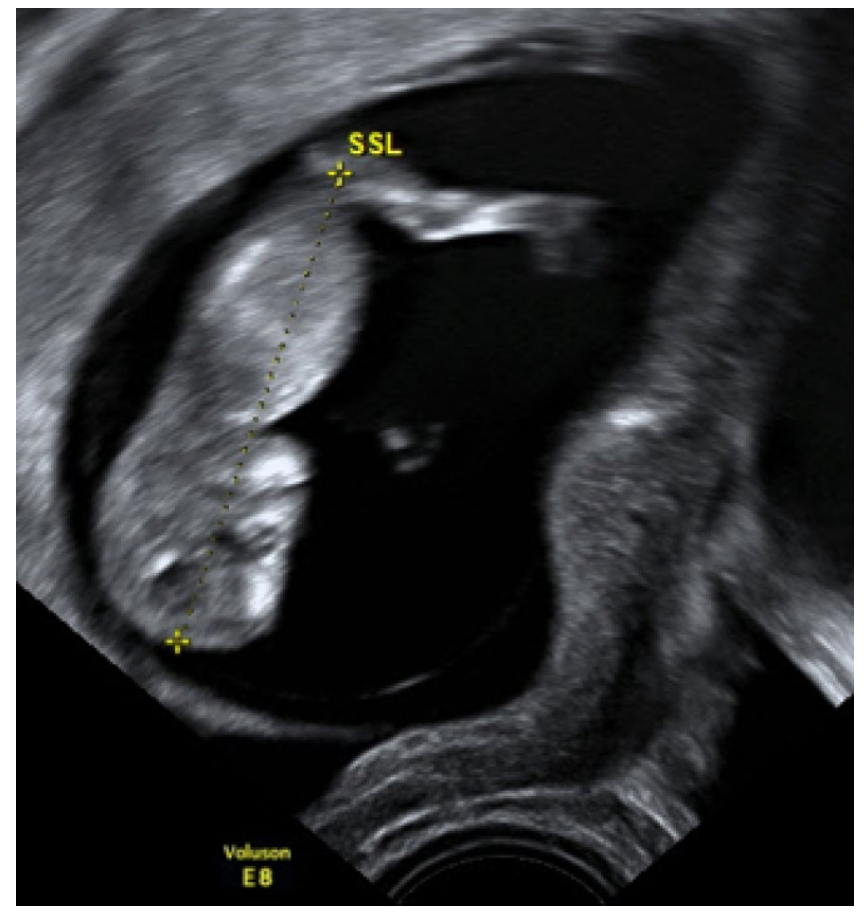

Abb. $6 \triangleleft 40,6 \mathrm{~mm}$ CRL, $10+5$ SSW nach Robinson und Hadlock, $10+4$ SSW nach Pexsters
3 Tage $(2,7 \mathrm{~mm})$ und überschätzen es in der 14. SSW um 2 Tage $(4,8 \mathrm{~mm})$ [6].

Die Scheitel-Steiß-Länge (SSL), auf Englisch „crown rump length“ (CRL), stammt aus der klassischen Embryologie der Carnegie-Klassifikation und wurde auf die Ultraschallbiometrie übertragen. Bereits derselbe Untersucher kann bei Messungen an derselben Patientin am selben Tag Ergebnisse dokumentieren, die einen Unterschied von 1,27 Tagen bedeuten, die Variabilität bei verschiedenen ausgebildeten und erfahrenen Untersuchern beträgt 1,37 Tage im Gestationsalter [7].

An den potenziellen Ungenauigkeiten bei der Festlegung des Gestationsalters, die an der natürlichen Variabilität der Zeitpunkte der Ovulation und der Implantation sowie der Langlebigkeit der Spermien liegen, kann auch der exakteste Ultraschall nichts ändern. Allerdings kann durch Beachtung möglicher Fehlerquellen und genaue Messung anhand der publizierten Standards verhindert werden, dass weitere Fehler dazu kommen [8].

\section{Biometrie in der frühen Frühschwan- gerschaft (•Tab. 2).}

1. Bei gerade erst sichtbarem Fruchtsack muss dieser in drei Ebenen gemessen werden und der Mittelwert dann zur
Berechnung herangezogen werden (- Abb. 1).

2. Hauptfehlerquelle ist die Messung des Embryos mit dem Dottersack, hier kann durch Einbeziehung des Dottersacks in die Messstrecke das tatsächliche Gestationsalter um bis zu eine Woche überschätzt werden (- Abb. 2).

3. Ein 3-D-Schall ist schön, die darin abgegriffenen Messstrecken sind allerdings wesentlich ungenauer als die von B-Mode-Aufnahmen (- Abb. 3 und 4).

4. Die Unterscheidung von Schädel und Steiß, die zephalokaudale Distinktion, ist ab der 7. SSW möglich (• Abb. 5).

Biometrie in der 10.-14. SSW (॰ Tab. 3).

1. Der Embryo ist nun sehr beweglich, er kann sich zusammenbeugen und strecken, hier ist mit einer gewissen Geduld eine Ruhephase des Embryos abzuwarten, in der er möglichst "gerade" ist, und dann erst zu messen (• Abb. 6).

2. Die in vielen Ultraschallgeräten mit der Messstrecke mitgelieferten Gestationsaltersberechnungen, immer auf den Tag genau, manchmal noch mit Stellen hinter dem Komma, sollten deaktiviert werden, da sie eine scheinbare Sicherheit suggerieren und die mitsehenden Schwangeren und ihre Angehörigen verwirren.

3. Bei IVF/ICSI/Kryoschwangerschaften den EGT nicht auf Basis der Biometrie später umdatieren! ARTEmbryonen wachsen genauso wie normal gezeugte [9]!

\section{Korrespondenzadresse}

Ao. Univ.-Prof. Dr. Christoph Brezinka Universitätsklinik für gynäkologische Endokrinologie und Reproduktionsmedizin, Medizinische Universität Innsbruck Anichstraße 35, 6020 Innsbruck, Österreich christoph.brezinka@i-med.ac.at

Funding. Open access funding provided by University of Innsbruck and Medical University of Innsbruck.

\section{Einhaltung ethischer Richtlinien}

Interessenkonflikt. C. Brezinka gibt an, dass kein Interessenkonflikt besteht.

Für diesen Beitrag wurden vom Autor keine Studien an Menschen oder Tieren durchgeführt. Für die aufgeführten Studien gelten die jeweils dort angegebenen ethischen Richtlinien.

Open Access. Dieser Artikel wird unter der Creative Commons Namensnennung 4.0 International Lizenz (http://creativecommons.org/licenses/by/4.0/deed. de) veröffentlicht, welche die Nutzung, Vervielfältigung, Bearbeitung, Verbreitung und Wiedergabe in jeglichem Medium und Format erlaubt, sofern Sie den/die ursprünglichen Autor(en) und die Quelle ordnungsgemäßnennen, einen Link zur Creative Commons Lizenz beifügen und angeben, ob Änderungen vorgenommen wurden.

\section{Literatur}

1. Mahendru AA, Wilhelm-Benartzi CS, Wilkinson IB, McEniery CM, Johnson S, Lees C (2016) Gestational length assignment based on last menstrual period, first trimester crown-rump length, ovulation, and implantation timing. Arch Gynecol Obstet 294:867-876. https://doi.org/10.1007/s00404016-4153-3

2. Johnson S, Shaw R, Parkinson P, Ellis J, Buchanan P, Zinaman M (2011) Home pregnancy testcompared to standard-of-care ultrasound dating in the assessment of pregnancy duration. Curr Med Res Opin 27:393-401. https://doi.org/10.1185/ 03007995.2010.545378

3. Bergsjo P, Denman DW III, Hoffman HJ, Meirik O (1990) Duration of human singleton pregnancy. A population-based study. Acta Obstet Gynecol Scand 69:197-207

4. WallerDK, Spears WD, GuY, Cunningham GC (2000) Assessing number-specific error in the recall of 
onset of last menstrual period. Paediatr Perinat Epidemiol 14:263-267

5. Robinson HP, Fleming JE(1975) A critical evaluation of sonar "crown-rump length" measurements. $\mathrm{Br} J$ Obstet Gynaecol 82:702-710

6. Pexsters A, Daemen A, Bottomley C, Van SD, De CL, DeMB, D'Hooghe T, Lees C, Timmerman D, BourneT (2010) New crown-rump length curve based on over 3500 pregnancies. Ultrasound Obstet Gynecol 35:650-655. https://doi.org/10.1002/uog.7654

7. Kagan KO, Hoopmann M, Baker A, Huebner M, Abele H, Wright $D$ (2012) Impact of bias in crownrump length measurement at first-trimester screening for trisomy 21. Ultrasound Obstet Gynecol 40:135-139. https://doi.org/10.1002/ uog. 11095

8. Blaas HG, Hasenohrl G, Staudach A (2018) Embryologie und Frühschwangerschaft. In: Gembruch U, Hecher K, Steiner H (Hrsg) Ultraschalldiagnostik in Geburtshilfe und Gynäkologie. Thieme, Stuttgart, S31-68

9. Eindhoven SC, van Uitert EM, Laven JS, Willemsen $\mathrm{SP}$, Koning AH, Eilers PH, Exalto N, Steegers EA, Steegers-Theunissen RP (2014) The influence of IVF/ICSI treatment on human embryonic growth trajectories. Hum Reprod 29:2628-2636. https:// doi.org/10.1093/humrep/deu271

10. Benson C, Doubilet P (2017) Fetal biometry and growth. In: Norton ME (Hrsg) Callen's ultrasonography in obstetrics and gynecology, 6. Aufl. Elsevier, Philadelphia, S118-131

Hinweis des Verlags. Der Verlag bleibt in Hinblick auf geografische Zuordnungen und Gebietsbezeichnungen in veröffentlichten Karten und Institutsadressen neutral.

\section{A. Platte \\ Das Ereignis der Geburt}

Medizinisches Wissen und Deutung des Geburtsaktes vom ausgehenden 18. bis zur Mitte des 19. Jahrhunderts

Bern, Schweiz: Peter Lang International Academic Publishers 2018, 1. Auflage, 270 S., (ISBN: 978-3631747261), Gebunden 49,95 EUR

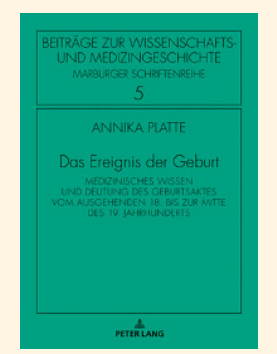

Annika Platte, Frauenärztin, tätig in Karlstadt, Schweden, legt ein medizinhistorisches Buch vor, welches detailliert und mit zahlreichen Quellenangaben versehen den Stand der Ansichten und des Wissens nachzeichnet, den man im 18. und 19.Jh. über die Entstehung menschlichen Lebens, Schwangerschaft und Geburt besaß.

Es ist erst 200 Jahre her, dass man darüber diskutierte, gelegentlich auch erbittert stritt, ob der Mensch präformiert, d.h. embryonal schon in der Anlage fertig geschaffen sei (Albrecht von Haller), oder epigenetisch, d.h. infolge einer den Körper gestaltenden, ausbildenden (und später auch regenerierenden) Kraft entstehe (Caspar Friedrich Wolff). Später standen Untersuchungen zur Atmung (Paul Zweifel) und Ernährung (William Preyer) des Feten im Vordergrund. Die Erkenntnis der Funktion von Plazenta und Fruchtwasser gewannen wissenschaftliches Interesse. Bezüglich der Auslösung der Geburt herrschten vom 17. bis zur Mitte des 18.Jh. verschiedene kontroverse Ansichten vor: Reifung der Plazenta, Dehnungseinflüsse auf die Uteruswand durch den wachsenden Fetus, muskuläre Reize, Hungerreaktion des Feten. Die muskuläre Beschaffenheit des Uterus stand danach im Mittelpunkt, als Alfred Kölliker die Existenz glatter neben der bekannten quergestreiften Muskulatur nachgewiesen hatte (1847). Von nun an konzentrierte sich die wissenschaftliche Debatte um das Zusammenwirken der muskulär-uterinen Geburtsphysiologie und der fötalen Autonomie.

Führend in der Schaffung eines wissenschaftlich begründeten Bildes wurden nun Friedrich Benjamin Osiander (1759-1822), Johann Christian Jörg (1779-1856), Carl Gustav Carus (1789-1869), Dietrich Wilhelm Heinrich
Busch (1788-1858) und Friedrich Wilhelm Johann Ignaz Scanzoni (1821-1891). Sie werden mit Biographien und ihren Beiträgen ausführlich dargestellt. Für die Geburtshilfe der Romantik, vertreten durch die genannten Forscher, bestand noch ein enges Verhältnis zur Naturphilosophie. Die Zusammenhänge mit der endokrinen Physiologie - der Begriff Hormone wurde erst 1905 von Ernest Starling (1866-1927) geprägt - verwandelte sich das Wissen um die Auslösung der Geburt grundlegend bis hin zu unseren heutigen Kenntnissen. Es ist das große Verdienst der Autorin, den langwierigen Weg nachgezeichnet zu haben, den unsere Kenntnisse bis heute genommen haben.

H. Ludwig (Basel) 